\title{
Efeito antiobesogênico da Pereskia grandifolia em ratos alimentados com dieta de cafeteria
}

\author{
Antiobesity effect Pereskia grandifolia in rats fed with cafeteria diet
}

Martha Elisa Ferreira de Almeida ${ }^{1}$, Danyany Marilia Costa Dias², José Antônio de Souza Cruz Ramos ${ }^{3}$

\section{RESUMO}

Introdução: A obesidade é um grave problema de saúde pública, cuja prevenção e tratamento têm sido pautados na utilização de alimentos saudáveis, incluindo as cactáceas do gênero Pereskia. Objetivo: Avaliar o efeito antiobesogênico da ora-pronóbis (Pereskia grandifolia) em ratos wistar alimentados com dieta de cafeteria. Material e Métodos: Os animais $(n=12)$ foram mantidos durante 8 semanas com a dieta de cafeteria a base de leite condensado, biscoito recheado de morango e óleo de milho. Posteriormente, eles foram distribuídos e mantidos durante 4 semanas nos grupos $\mathrm{C}$ (dieta e recebimento semanal de $4 \mathrm{~mL}$ de água, via gavagem) e $S$ (dieta e recebimento de $4 \mathrm{~mL}$ de suco das folhas de ora-pro-nóbis, via gavagem). Avaliou-se o peso corporal e dos órgãos; o consumo alimentar; o peso das fezes e a Digestibilidade Aparente da dieta de cafeteria; os Índices de Massa Corporal (IMC), de Gordura Visceral (IGV) e Gordura Epididimal (IGE); e a quantidade de tecido adiposo (visceral e epididimal). Os dados foram comparados pelo Teste Mann-Whitney a significância de 5\%. Resultados: Não houve diferença estatística quanto ao peso corporal, dos órgãos e das fezes; da Digestibilidade Aparente e do Índice de Gordura Visceral. O grupo S apresentou um menor consumo alimentar apenas na semana 2 e um menor IMC no final do experimento. Conclusão: $O$ suco das folhas de Pereskia grandifolia não reduziu o peso corporal dos animais, entretanto diminuiu a adiposidade.

Descritores: Ora-pro-nóbis. Compostos bioativos. Índice de Gordura.

\section{ABSTRACT}

Introduction: Obesity is a serious public health problem whose prevention and treatment has been based on the use of healthy foods, including cacti of the genus Pereskia. Objective: To evaluate the antiobesity effect ora-pro-nobis (Pereskia grandifolia) in wistar rats fed cafeteria diet. Material and Methods: Animals $(n=12)$ were kept for 8 weeks with cafeteria diet the basis of condensed milk, cookie filled with strawberry and corn oil. Subsequently, they were distributed and kept for 4 weeks in the groups C (diet and weekly receipt of $4 \mathrm{~mL}$ of water, by gavage) and S (diet and receiving $4 \mathrm{~mL}$ leaf juice of ora-pro-nobis, by gavage). Was evaluated body weight and organ; food consumption; the stool weight and Apparent Digestibility of cafeteria diet; the Body Mass Index (BMI), of Visceral Fat (IGV) and Epididymal Fat (IGE); and the amount of adipose tissue (visceral and epididymal). Data were compared using the Mann-Whitney Test the significance of $5 \%$. Results: There was no statistical difference in body weight, organ and stool; the Apparent Digestibility and Visceral Fat Index. The S group had a lower food consumption only at week 2 and lower BMl at the end of the experiment. Conclusion: The juice Pereskia grandifolia sheet did not reduce the body weight of the animals, however decreased adiposity.

Descriptors: Ora-pro-nobis. Bioactive compounds. Fat Index.

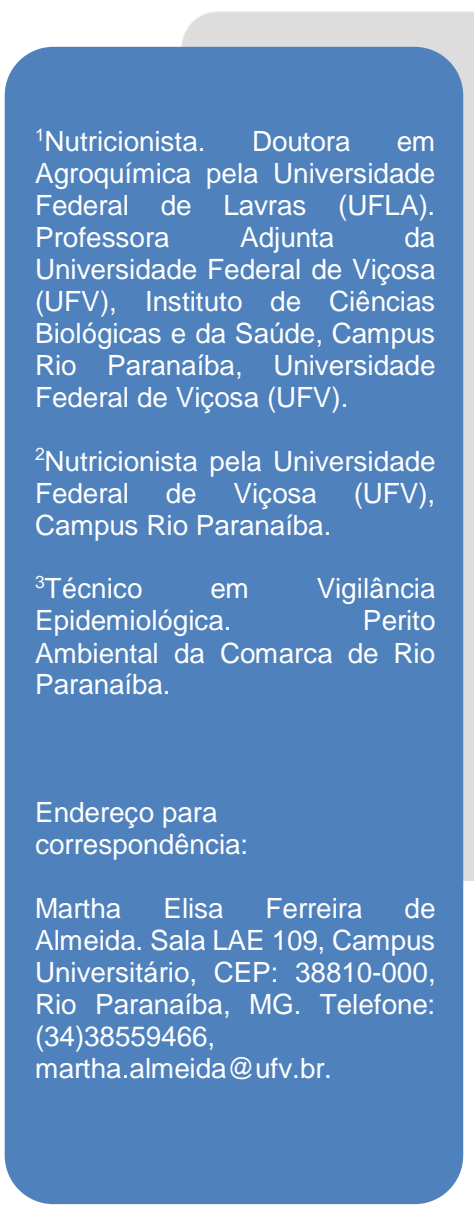




\section{INTRODUÇÃOO}

A obesidade é uma doença crônica não transmissível, caracterizada pelo excesso de gordura corporal ${ }^{1}$. De origem multifatorial, ela pode incluir diversos fatores como psicológicos, socioeconômicos, biológicos e ambientais com ênfase para a ingestão alimentar. Esta patologia é um problema mundial que atinge todos os níveis socioeconômicos, idades e etnias, e gera grandes prejuízos aos cofres públicos decorrentes das condições associadas a ela, como a hipertensão arterial sistêmica; as dislipidemias; o diabetes mellitus tipo 2; a doença vascular arteriosclerótica; os problemas psiquiátricos com episódios depressivos, a baixa-estima e a alteração dismórfico corporal².

A população mundial tem apresentado várias modificações no perfil nutricional, o que pode ser atribuído a um processo conhecido como transição nutricional, no qual há uma alteração da desnutrição para uma maior prevalência do sobrepeso e da obesidade 2 . De acordo com dados da Pesquisa de Orçamentos Familiares ${ }^{3}$ de 2008-2009, o excesso de peso no Brasil atingia $49 \%$ da população acima de 20 anos, e a obesidade acometia $14,8 \%$. Quando se tratava do excesso de peso $^{3}$, a prevalência era maior em homens $(50,1 \%)$ em comparação às mulheres $(48 \%)$, no entanto a obesidade estava mais presente no gênero feminino (16,9\%) quando comparada ao masculino (12,5\%).

O tratamento do excesso de peso é complexo, e deve ser baseado na prescrição de dietas que proporcionem um aumento na ingestão de alimentos in natura como frutas, verduras e hortaliças, que apresentam nutrientes importantes ao metabolismo, com destaque para alguns compostos fitoquímicos que possuem atividade antiobesidade 4 .

A ora-pro-nóbis é uma planta da família Cactaceae, gênero Pereskia, que vem sendo utilizada na medicina popular, entretanto, ainda tem sido pouco consumida pela população, em decorrência da falta de informações quanto ao seu valor nutricional ${ }^{4}$. Na Malásia as folhas da Pereskia grandifolia são utilizadas no tratamento do câncer, hipertensão arterial sistêmica, diabetes mellitus tipo 2, reumatismo e inflamação, dor de cabeça, dermatite atópica e hemorroidas ${ }^{5}$, dor gástrica e úlcera ${ }^{6}$; enquanto na Índia ela é adotada para o controle do edema? .

Já foram identificados vários fitoquímicos na Pereskia grandifolia, com destaque para duas saponinas do ácido oleanoico ${ }^{7}$; taninos e flavonoides ${ }^{8}$; alcaloides ( $p$-metoxi- $\beta$-hidroxi$\beta$-fenetillamina, 3-metohoxitiramina e tiramina) ${ }^{9}$; o $\beta$-sitosterol, vitamina $E$, fitona e o 2,4ditert-butilfenol ${ }^{10}$. 
O objetivo do estudo foi avaliar o efeito antiobesogênico da Pereskia grandifolia em ratos wistar alimentados com dieta de cafeteria.

\section{MATERIAIS E METODOS}

O experimento foi desenvolvido de acordo com os Princípios Éticos na Experimentação Animal, segundo a Lei ${ }^{11}$ oo 11.794 de 8 de outubro de 2008, tendo sido o projeto aprovado pela Comissão de Ética no Uso de Animais (CEUA) da Universidade Federal de Viçosa (UFV), protocolo no 56/2015.

\section{Ensaio biológico}

Realizou-se o experimento durante um período de 12 semanas no Laboratório de Nutrição Experimental da Universidade Federal de Viçosa, Campus Rio Paranaíba (MG), utilizando 12 ratos wistar machos, com peso inicial médio de $60,43 \pm 3,50 \mathrm{~g}$. Os animais foram mantidos em gaiolas individuais, sob temperatura ambiente de $21{ }^{\circ} \mathrm{C}$, ciclo claro/escuro de 12 horas e recebimento de dieta e água, ambos ad libitum.

\section{Preparo das dietas}

Utilizou-se leite condensando (46\%), biscoito recheado sabor morango (46\%) e óleo de milho (8\%), sendo a dieta composta de $57,5 \mathrm{~g}$ de carboidratos $(50,10 \%) ; 5,96 \mathrm{~g}$ de proteínas $(5,19 \%)$; e $22,8 \mathrm{~g}$ de lipídios $(44,70 \%)$, totalizando $459,04 \mathrm{kcal} \cdot 100 \mathrm{~g}^{-1}$ de dieta. Uma dieta padrão para roedores da marca Biobase $\AA$ possui $300 \mathrm{kcal} \cdot 100 \mathrm{~g}^{-1}$ de dieta, sendo composta de $58,67 \%$ de carboidratos; $29,33 \%$ de proteínas e $12,00 \%$ de lipídios.

Preparou-se o suco das folhas de ora-pro-nóbis (Pereskia grandifolia) com 100g de folhas picadas, previamente higienizadas, e liquidificadas em $900 \mathrm{~mL}$ de água, sendo que 0 mesmo foi coado para a administração nos animais, via gavagem, com agulha curva com ponta tipo bola.

Os animais permaneceram por um período de 8 semanas recebendo a dieta de cafeteria e água, ambos ad libitum. Após este período foram distribuídos em 2 grupos: Controle ( $C$ - dieta de cafeteria e a administração semanal de $4 \mathrm{~mL}$ de água, via gavagem); e o Grupo do suco das folhas de ora-pro-nóbis (S - dieta de cafeteria e a administração semanal de $4 \mathrm{~mL}$ do suco das folhas de ora-pro-nóbis, via gavagem). O peso médio dos 
animais no início desta fase de utilização do suco de folha de ora-pro-nóbis era de $151,58 \pm 18,81 \mathrm{~g}$. Realizou-se a gavagem nos dias de segunda-feira e sexta-feira de 12:00 as 14:00 horas, sendo que em cada dia administrou-se apenas $2 \mathrm{~mL}$ de água ou suco das folhas de ora-pro-nóbis para evitar desconforto gástrico aos animais. Utilizou-se gel anestésico (Lidogel) na agulha de gavagem.

Prepararam-se as dietas semanalmente na quantidade de $2 \mathrm{~kg}$, e conservou-as sob refrigeração para minimizar a oxidação lipídica. Calculou-se a ingestão alimentar diária pela diferença entre a dieta ofertada e suas sobras. O peso corporal e a distância nasoanal foram medidos semanalmente ${ }^{12}$ para o cálculo do Índice de Massa Corporal (IMC), que é obtido através da razão: peso corporal $(\mathrm{g}) /$ comprimento $(\mathrm{cm}) \times$ comprimento $(\mathrm{cm})$.

\section{Coleta das fezes}

Coletaram-se as fezes semanalmente, no período de utilização do suco das folhas de ora-pro-nóbis, entre os dias de segunda-feira e quarta-feira para o cálculo da Digestibilidade Aparente ${ }^{13}$, conforme a fórmula descrita a seguir:

Digestibilidade Aparente $=$ [(quantidade ingerida de alimentos- quantidade excretada de fezes)/quantidade ingerida de alimentos]

\section{Sacrifício e análise tecidual}

Ao final do experimento aplicou-se em cada animal o anestésico geral intravenoso (Propofol) na dosagem de $10 \mathrm{~mL} \cdot \mathrm{kg}^{-1}$ de peso corporal, conforme descrito na Resolução Normativa ${ }^{14} \mathrm{n}$ - 13 de 20 de setembro de 2013. Retirou-se aproximadamente $4 \mathrm{~mL}$ de sangue por punção cardíaca, que promoveu a morte do animal por exsanguinação.

O fígado, os rins e o baço foram retirados por laparotomia mediana, lavado em solução salina $(0,9 \%)$ e pesado. Coletaram-se os tecidos adiposos (visceral e epididimal) para o cálculo do Índice de gordura (IG). Determinou-se o Índice de Gordura Visceral ${ }^{15}$ através da relação entre o peso da gordura visceral (GV) e o peso corporal final (PCF) do animal, onde: IGV = (GV/PCF) X 100. Obteve-se o Índice de Gordura Epididimal pela relação entre o peso da gordura epididimal (GE) e o peso corporal final (PCF) do animal, onde: IGE = (GE/PCF) X 100. 


\section{Análise estatística}

Os valores são representados como média (M) e desvio-padrão (DP). Realizou-se a comparação das médias pelo Teste de Mann-Whitney no programa Statistical Package for Social Sciences (SPSS), versão 20.0, a 5\% de significância.

\section{RESULTADOS E DISCUSSÁO}

Não houve diferença estatística $(p>0,05)$ do peso corporal entre os grupos durante 0 experimento (Figura 1A), assim como no consumo alimentar (Figura 1B) nas semanas 1, 3 e 4, respectivamente. Somente na semana 2, o grupo $S$ que recebeu a suco das folhas de ora-pro-nóbis apresentou um menor consumo alimentar $(p<0,05)$. $O$ grupo $S$ apresentou um menor ganho de peso médio $(p<0,05)$ durante o período de utilização do suco das folhas de ora-pro-nóbis ( $\mathrm{S}=20,33 \pm 10,26 \mathrm{~g} ; \mathrm{C}=30,17 \pm 6,41 \mathrm{~g}$ ).

A

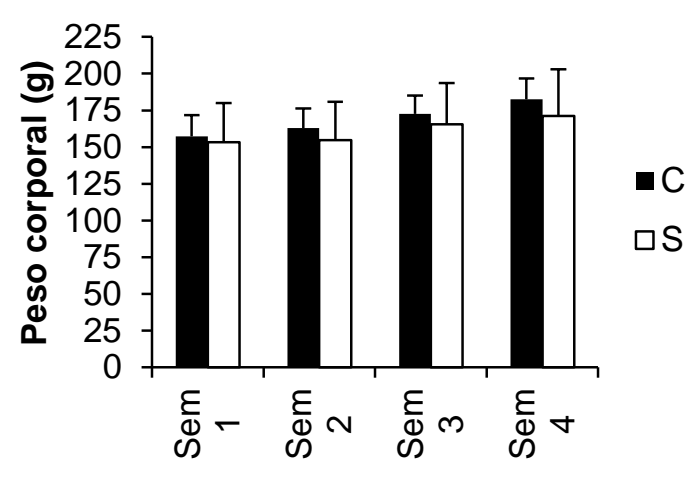

B

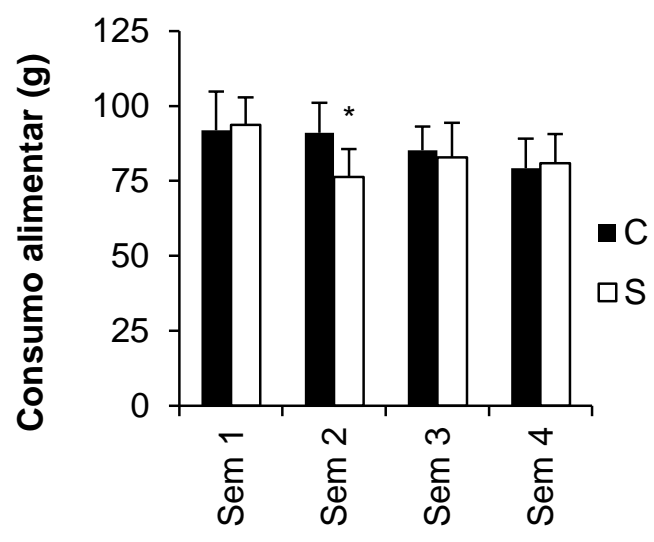

Figura 1 Média e desvio padrão do peso corporal (A) e do consumo alimentar (B) dos animais dos grupos controle $(C)$ e suco das folhas de ora-pro-nóbis (S), durante o período de utilização da Pereskia grandifolia. Rio Paranaíba, MG, 2016. Sem = semana; *Significativo a 5\% pelo Teste de Mann-Whitney.

Pesquisas com modelos animais e dietas de cafeteria têm sido realizadas, devido ao fato dessas dietas representarem o cotidiano alimentar de alguns humanos ${ }^{16}$, bem como promoverem o excesso de peso corporal total ou de tecido adiposo. Assim, estudos com 
plantas na forma extratos, soluções ou farinhas têm sido realizados ${ }^{17,18}$, visando a redução do peso corporal ou da quantidade de tecido adiposo. Entretanto, a farinha da taioba ${ }^{19}$ aumentou o peso corporal de ratos wistar alimentados com dieta de cafeteria (hipercalórica e hiperlipídica).

Não houve diferença estatística quanto o peso corporal entre os grupos deste estudo, demonstrando que o suco das folhas de ora-pro-nóbis não exerceu efeito de redução do peso corporal total nos animais. Ao avaliarem o efeito do extrato de metanol da Pereskia grandifolia em camundongos (machos e fêmeas) de cinco semanas de idade durante 14 dias, observou-se que eles ganharam peso e não apresentaram sinais clínicos de toxicidade em nenhuma das doses utilizadas ${ }^{5}$. Houve diminuição do peso corporal de ratos alimentados com dieta hiperlipídica e extrato de algas vermelhas (Plocamium telfairiae) ${ }^{20}$, e com farinha de ora-pro-nóbis ${ }^{12}$ (Pereskia grandifolia) na quantidade de 5 e 10\%. Entretanto, não se identificou diferença no peso corporal dos ratos que receberam dieta padrão e solução aquosa de yacon (Smallanthus sonchifolius) quando comparados aos seus controles que ingeriram água ${ }^{21}$; nos animais que receberam, via gavagem, o extrato de café do mato (Cordia ecalyculata Vell) nas doses de 20, 100 e $400 \mathrm{mg}^{\mathrm{kg}}{ }^{-1}$ de peso corpóreo ${ }^{22}$; naqueles alimentados com dieta padrão e solução, ad libitum, de chá mate (Ilex paraguariensis) ${ }^{23}$; e nos tratados com $2 \mathrm{~mL}$, via gavagem, de extrato de chá verde (Camelia sinensis) ${ }^{18}$.

O grupo que recebeu o suco de ora-pro-nóbis apresentou um menor IMC $(0,45 \pm 0,04)$ que o grupo controle $(0,48 \pm 0,02)$, assim como observado no estudo ${ }^{12}$ com a farinha da Pereskia grandifolia.

O consumo alimentar semanal dos grupos $\mathrm{C}$ e $\mathrm{S}$ diferiram estatisticamente apenas na semana 2 do experimento, sendo que no estudo ${ }^{12}$ com 5 e 10\% da farinha da folha de ora-pro-óbis (Pereskia grandifolia), os animais apresentaram um menor consumo alimentar durante as 4 semanas de utilização desta cactácea. O suco de laranja vermelha (Citrus grandis) promoveu um menor consumo alimentar dos ratos previamente alimentados com a dieta hiperlipídica ${ }^{24}$, entretanto, não identificou diferença estatística nos grupos tratados com dieta hiperlipídica e extrato de chá mate (llex paraguariensis) ${ }^{25}$,extrato aquoso de alho

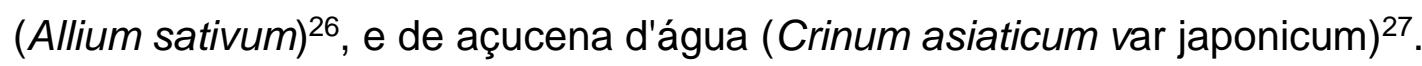

O peso dos órgãos avaliados não diferiu entre os grupos (Tabela 1), assim como observado no estudo ${ }^{28}$ com extrato etanólico de raiz-de-lótus (Nelumbo nucifera). Divergindo de outros estudos onde o peso do fígado foi menor nos grupos alimentados com 
5 e $10 \%$ de farinha de ora-pro-nóbis (Pereskia grandifolia) ${ }^{12}$, e maior naqueles que ingeriram o extrato etanólico de malmequer (Calendula officinales) ${ }^{29}$.

Tabela 1 Média e desvio padrão do peso dos órgãos (fígado, rins e baço), do tecido adiposo (visceral e epididimal) e do Índice de Gordura (Visceral e Epididimal) dos grupos controle (C) e suco das folhas de ora-pro-nóbis (S), durante o período de utilização da Pereskia grandifolia. Rio Paranaíba, MG, 2016.

\begin{tabular}{lcc}
\hline \multirow{2}{*}{ Parâmetro avaliado } & \multicolumn{2}{c}{ Grupos } \\
\cline { 2 - 3 } & $\mathbf{C}$ & $\mathbf{S}$ \\
\hline Fígado (g) & $8,80 \pm 0,48$ & $8,78 \pm 0,26$ \\
Rins $(\mathrm{g})$ & $1,61 \pm 0,16$ & $1,58 \pm 0,11$ \\
Baço (g) & $0,34 \pm 0,04$ & $0,36 \pm 0,06$ \\
TAV (g) & $2,41 \pm 0,17$ & $1,88 \pm 0,69^{*}$ \\
TAE (g) & $2,37 \pm 0,30$ & $1,87 \pm 0,31^{*}$ \\
IGV & $1,33 \pm 0,11$ & $1,09 \pm 0,34$ \\
IGE & $1,29 \pm 0,10$ & $1,11 \pm 0,16^{*}$ \\
\hline
\end{tabular}

TAV = Tecido Adiposo Visceral; TAE = Tecido Adiposo Epididimal; IGV = Índice de Gordura Visceral; IGE = Índice de Gordura Epididimal; *Significativo a 5\% pelo Teste de MannWhitney para o parâmetro avaliado na linha.

Os animais que receberam o suco das folhas de ora-pro-nóbis (Pereskia grandifolia) apresentaram menores quantidades de tecido adiposo visceral $(21,99 \%)$ e epididimal $(21,10 \%)$ que o grupo controle, diferindo do estudo ${ }^{12}$ com a farinha de ora-pro-nóbis (5 e $10 \%$ ), no qual os ratos previamente alimentados com dieta de cafeteria reduziram o consumo alimentar, o peso corporal e os lipídios hepáticos, mas não alteraram significativamente os lipídios corporais totais.

Identificou-se uma menor quantidade de tecido adiposo epididimal em ratos wistar pré-alimentados com dieta hiperlipídica e posteriormente administrado chá mate (Ilex paraguariensis), via gavagem ${ }^{21}$, na concentração de $1 \mathrm{~g} \cdot 100 \mathrm{~g}^{-1}$; e naqueles que receberam o extrato etanólico de espinheiro marítimo (Sea buckthorm) nas doses de 500 e 1000mg . $\mathrm{kg}^{-1}$ de peso corporal ${ }^{30}$. 
Houve diminuição do tecido adiposo pela inibição da lipase pancreática em ratos alimentados com dieta hiperlipídica e extrato de flor-de-lótus (Nelumbo nucifera) ${ }^{31}$, bem como pelas saponinas do ginseng (Panax ginseng $)^{32}$.Sugere-se que as saponinas do ácido oleanoico, já identificadas na Pereskia grandifolia ${ }^{7}$, podem ter exercido efeito antiobesidade nos animais deste estudo com o suco das folhas de ora-pro-nóbis.

O excesso de tecido adiposo, principalmente o visceral, poderá promover o surgimento da doença arterial coronariana, hipertensão arterial sistêmica, diabetes mellitus tipo 2, dislipidemias, distúrbios da coagulação, hiperuricemia e microalbuminúria, e alguns tipos de cânceres. Assim, a redução deste tecido diminui significativamente as chances do surgimento dessas doenças ou seus sintomas ${ }^{33,34}$.

Não houve diferença no Índice de Gordura Visceral (Tabela 1), divergindo do estudo com ratas ovariectomizadas que receberam $5 \%$ de extrato de raiz da vida eterna (Panax ginseng), onde se identificou um maior Índice de Gordura Visceral ${ }^{35}$, enquanto os camundongos alimentados com dieta hiperlipídica e extrato aquoso de folhas de bora-bora (Ligustrum robustum Blume) apresentaram um menor Índice de Gordura Visceral ${ }^{36}$.

Os animais que receberam o suco das folhas de ora-pro-nóbis apresentaram um menor Índice de Tecido Adiposo Epididimal, assim como observado em ratos alimentados com dieta hiperlipídica tratados com extrato aquoso de folhas de guajuru (Chrysobalanus icaco L.) $)^{37}$, e nos camundongos C57BL/6J alimentados com dieta hiperlipídica, juntamente com o extrato de tomate verde (Solanum lycopersicum) ${ }^{38}$. Com base nos dados deste estudo, observou-se que a Pereskia grandifolia reduziuestatisticamente apenas o Índice de Gordura Epididimal.

O peso das fezes não diferiu entre os grupos (Figura 2A), assim como observado entre os ratos wistar tratados com extrato aquoso de chá mate (llex paraguariensis) ${ }^{23}$. $\mathrm{O}$ suco das folhas de ora-pro-nóbis diminuiu a adiposidade, entretanto não promoveu alteração da Digestibilidade Aparente quando comparado ao grupo controle (Figura 2B), assim como observado nos camundongos C57BL/6J alimentados com dieta hiperlipídica, juntamente com o extrato de tomate verde (Solanum lycopersicum $)^{38}$.

A
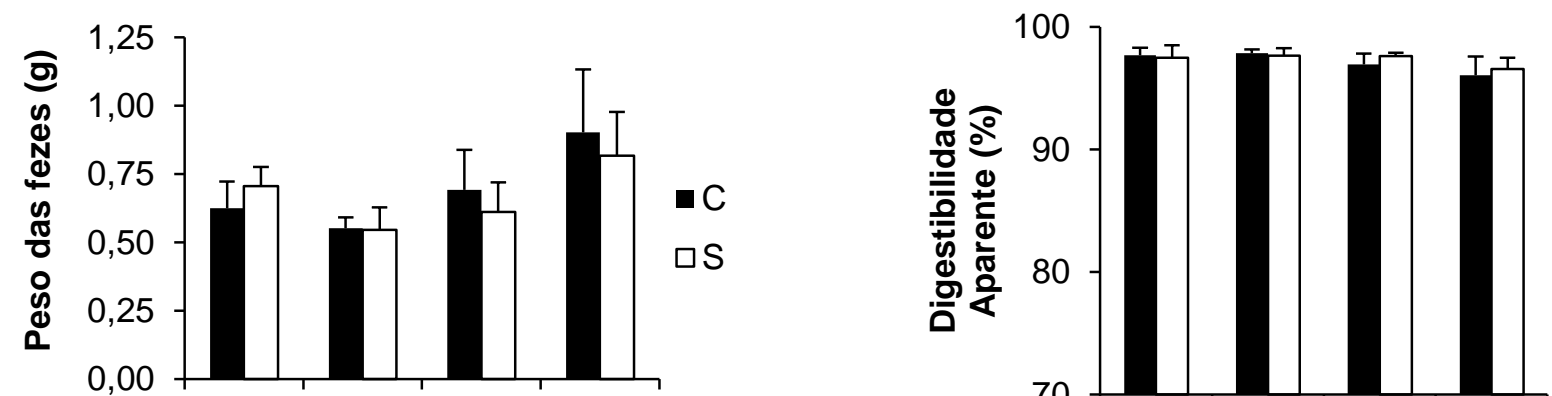
Figura 2- Média e desvio padrão do peso das fezes (A) e da Digestibilidade Aparente (B) dos grupos controle (C) e suco das folhas de ora-pro-nóbis (S), durante o período de utilização da Pereskia grandifolia. Rio Paranaíba, MG, 2016. Sem = semana.

\section{CONSIDERAÇÓES FINAIS}

O suco das folhas de ora-pro-nóbis não alterou o peso corporal total, e o consumo alimentar na maioria das semanas estudadas, o peso dos órgãos e a Digestibilidade Aparente da dieta de cafeteria, entretanto apresentou efeito antiobesidade, pois reduziu a adiposidade visceral e epididimal, sugerindo que tal efeito tenha sido propiciado por algum fitoquímico presente nesta cactácea.

Como limitação deste estudo destaca-se a utilização do suco das folhas da Pereskia grandifolia somente durante 4 semanas, sugerindo que pesquisas posteriores sejam realizadas com uma maior duração do tratamento e utilizem outros modelos animais.

\section{REFERÉNCIAS}

1. Pinho $\mathrm{CPS}$, et al. Prevalência e fatores associados à obesidade abdominal em indivíduos na faixa etária de 25 a 59 anos do Estado de Pernambuco, Brasil. Cad Saúde Pública 2013;29(2):313-324.

2. Corso ACT, et al. Fatores comportamentais associados ao sobrepeso e à obesidade em escolares do Estado de Santa Catarina. Ver. Bras Estud Popul 2012;29(1):117-131.

3. Instituto Brasileiro de Geografia e Estatística (IBGE). Pesquisa de Orçamentos Familiares Antropometria e estado nutricional de crianças, adolescentes e adultos no Brasil, p. 54-57, 2009. [acesso em 06 maio 2019]. Disponível em: http://biblioteca.ibge.gov.br/visualizacao/livros/liv45419.pdf.

4. Almeida MEF, Corrêa AD. Utilização de cactáceas do gênero Pereskia na alimentação humana em um município de Minas Gerais. Cienc Rural 2012;42(4):751-756.

5. Sim KS, Sri Nurestri AM, Sinniah SK, Kim KH, Norhanom AW. Acute oral toxicity of Pereskia bleo and Pereskia grandifolia in mice. Pharmacogn Mag 2010;6(21):67-70.

6. Goh KL. Malaysian herbaceous plants millennium. Advanco Press: Malaysia. 2000.

7. Sahu NP, Benerji N, Chakravarti RN. New saponin of oleanolic acid from Pereskia grandifolia. Phytochem 1974;13(2):529-530. 
8. Turra AF, Marçal FJB, Baretta IP, Takemura OS, Laverde Jr A.Avaliação das propriedades antioxidantes e susceptibilidade antimicrobiana de Pereskia grandifolia Haworth (Cactaceae). Arq Ciênc Saúde da Unipar 2007;11(1):9-14.

9. Doetsch PW, Cassady JM, Mclaughlin JL. Cactus alkaloids. XL. Identification of mescaline and other $\beta$-fenethylamines in Pereskia, Pereskiopsis and Islaya by use of fluorescamine conjugates. J Chromatogr 1980;189(1):79-85.

10. Sri Nurestri AM, Sim KS, Norhanom AW. Phytochemical and cytotoxic investigations of Pereskia grandifolia Haw. (Cactaceae) leaves. J Biol Sci 2009;9(5):488-493.

11. Brasil. Ministério da Saúde. Lei oo 11.794, de 8 de outubro de 2008. Procedimentos para o uso científico de animais. Diário Oficial da República Federativa do Brasil, Brasília, DF, 9 out. 2008. [acesso em 06 maio 2019]. Disponível em: http://www.planalto.gov.br/ccivil_03/_ato20072010/2008/lei/l11794.htm.

12. Almeida MEF, Simão AA, Corrêa AD, Fernandes RVB. Improvement of physiological parameters of rats subjected to hypercaloric diet, with the use of Pereskia grandifolia (Cactaceae) leaf flour. Obes Res Clin Pract 2016;10(6):701-709.

13. Maynard LA, Loosli JK. Nutrição Animal. Rio de Janeiro: USAID, 1966.

14. Brasil. Resolução Normativa, no 13 de 20 de setembro de 2013 - CONCEA. Baixa as Diretrizes da Prática de Eutanásia do Conselho Nacional de Controle de Experimentação Animal. [acesso em 06 maio 2019]. Disponível em:http://www.mct.gov.br/upd_blob/0228/228451.pdf.

15. Leite MAG. Influência do tanino sobre a concentração de colesterol em ratos submetidos à ingestão de dieta rica em lipídeos. Dissertação [Mestrado em Alimentos e Nutrição], Faculdade de Ciências Farmacêuticas. Universidade Estadual Paulista. Araraquara; 2003.

16. Rosini TCS, Silva ASR, Moraes C. Obesidade induzida por consumo de dieta: modelo em roedores para o estudo dos distúrbios relacionados com a obesidade. Rev Assoc Med Bras 2012;58(3):383387.

17. Sung YY, Kim DS, Kim HK. Akebia quinata extract exerts anti-obesity and hypolipidemic effects in high-fat diet-fed mice and 3T3-L1 adipocytes. J Ethnopharmacol 2015;168:17-24.

18. Vera-Cruz M, Nunes E, Mendonça L, Chaves É, Fernandes MLLA. Efeito do chá verde (Camelia sinensis) em ratos com obesidade induzida por dieta hipercalórica. J Bras Patol Med Lab2010;46(5):407-413.

19. Silva $L T$, et al. Efeito da ingestão de farinha de taioba sobre o peso corporal de ratos previamente alimentados com óleo de soja e banha suína. SaBios: Rev Saúde e Biol 2015;10(2):27-34.

20. Kang MC, Kang N, Ko SC, Kim YB, Jeon YJ. Anti-obesity effects of seaweeds of Jeju Island on the differentiation of 3T3-L1 preadipocytes and obese mice fed a high-fat diet. Food Chem Toxicol 2016;90:36-44.

21. Oliveira LA, Costa TMB, Oliveira LRA, Ferreira JF, Navarro AM. Repostas glicêmicas de ratos diabéticos recebendo solução aquosa de yacon. Alim Nutr 2009;20(1):61-67.

22. Campos MC. Avaliação do potencial tóxico reprodutivo de extrato de Cordia ecalyculata Vell, em ratos wistar. Dissertação [Mestrado em Biociências], Faculdade de Ciências e Letras de Assis, Universidade Estadual Paulista. Assis; 2014. 
DOI 10.18606/2318-1419/amazonia.sci.health.v7n2p43-54
Almeida MEF, Dias, DMC, Ramos, JASC.

Efeito antiobesogênico da Pereskia grandifolia em ratos alimentados com dieta de cafeteria

23. Pedroso GL, Mendes RH, Persh K, Jahn MP, Kucharski LC. Efeito do extrato aquoso de Ilex paraguariensis sobre o metabolismo de ratos machos. Rev HCPA 2010;30(3):241-246.

24. Massafera G. Efeito protetor do suco de laranja vermelha e dieta hiperlipídica nos parâmetros fisiológicos e marcadores bioquímicos emratos submetidos à dieta hiperlipídica. Tese [Doutorado], Universidade Estadual Paulista "Júlio de Mesquita Filho", Faculdade de Ciências Farmacêuticas. Araraquara; 2014.

25. Gomes LF. Efeito da erva mate (Ilex paraguaiensis) sobre o metabolismo de ratos tratados com dieta hiperlipídica. Dissertação [Mestrado em Ciências Biológicas], Universidade Federal do Rio Grande do Sul. Porto Alegre; 2012.

26. Joo H, Kim CY, Kim IH, Kim Y. Anti-obesity effects of hot water extract and high hydrostatic pressure extract of garlic in rats fed a high-fat diet. Food Chem Toxicol 2013; 55:100-105.

27. Jeong YJ, et al. Anti-obesity effect of Crinum asiaticum var. japonicum Baker extract in high-fat dietinduced and monogenic obese mice. Biomed Pharmacother 2016; 82:35-43.

28. You JS, Lee YJ, Kim KS, Kim SH, Chang KJ. Ethanol extract of lotus (Nelumbo nucifera) root exhibits an anti-adipogenic effect in human pre-adipocytes and anti-obesity and anti-oxidant effects in rats fed a high-fat diet. Nutr Res 2014;34(3):258-267.

29. Fontes GG. Efeito de formulação fitoterápica contendo Calendula officinalis no metabolismo lipídico em ratos wistar alimentados com dieta de cafeteria. Dissertação [Mestrado em Bioquímica Agrícola], Universidade Federal de Viçosa. Viçosa; 2015.

30. Pichiah PB, Moon HJ, Park JE, Moon YJ, Cha YS. Ethanolic extract of seabuckthorn (Hippophae rhamnoides $\mathrm{L}$ ) prevents high-fat diet-induced obesity in mice through down-regulation of adipogenic and lipogenic gene expression. Nutr Res 2012;32(11):856-864.

31. Ono Y, Hattori E, Fukaya Y, Imai S, Ohizumi Y. Anti-obesity effect of Nelumbo nucifera leaves extract in mice and rats. JEthanopharmacol2006;106(2):238-244.

32. Karu N, Reifen R, Kerem Z. Weight gain reduction in mice fed Panax ginseng saponin, a pancreatic lipase inhibitor. J Agric Food Chem 2007;55(8):2824-2828.

33. Romero CEM, Zanesco A. O papel dos hormônios leptina e grelina na gênese da obesidade. Rev Nutr 2006;19(1):85-91.

34. Ribeiro Filho FF, Mariosa LS, Ferreira SRG, Zanella MT. Gordura visceral e síndrome metabólica: mais que uma simples associação. Arq Bras Endocrinol Metab 2006;50(2):230-238.

35. Lee H, Choi J, Shin SS, Yoon M. Effects of Korean red ginseng (Panax ginseng) on obesity and adipose inflammation in ovariectomized mice. J Ethnopharmacol 2016; 178:229-237.

36. Yang RM, et al. Anti-obesity effect of total phenylpropanoid glycosides from Ligustrum robustum Blume in fatty diet-fed mice via up-regulating leptin. J Ethnopharmacol 2015; 169:459-465.

37. White PA, et al. Aqueous extract of Chrysobalanus icaco leaves, in lower doses, prevent fat gain in obese high-fat fed mice. J Ethnopharmacol 2016; 179:92-100.

38. Choi KM, et al. Green tomato extract attenuates high-fat-diet-induced obesity through activation of the AMPK pathway in C57BL/6 mice. J Nutr Biochem 2013;24(1):335-342. 